1 Universidade do Vale do Taquari (Univates) Lajeado (RS), Brasil. cgotlermedeiros@gmail.com

\section{Processos de encaminhamento a serviços especializados em cardiologia e endocrinologia pela Atenção Primária à Saúde}

\author{
Referral processes to services specialized in cardiology and \\ endocrinology for Primary Health Care
}

Sérgio Vieira Bernardino Junior ${ }^{\mathbf{1}}$, Cássia Regina Gotler Medeiros ${ }^{\mathbf{1}}$, Camila Furtado de Souza ${ }^{\mathbf{1}}$, Jordana Kich', Alessandro Menna Alves', Luís César de Castro'

DOI: 10.1590/0103-1104202012608

RESUMO Este estudo objetivou analisar encaminhamentos de pessoas a serviços de cardiologia e endocrinologia pelos médicos da Atenção Primária à Saúde (APS) de um município do Rio Grande do Sul. Estudo transversal com questionários a 25 médicos da APS, 2 cardiologistas, 2 endocrinologistas e 1 médico regulador. A análise estatística foi realizada no SPSS, sendo as variáveis contínuas apresentadas como média ou mediana; e as variáveis categóricas, como frequência absoluta e relativa. Entre os médicos da APS, 96\% conhecem, 84\% utilizam protocolos de encaminhamento e $92 \%$ encaminham os pacientes principalmente para manejo de doenças complicadas que necessitam de avaliação do especialista. Entre os especialistas, $50 \%$ conhecem os protocolos, e todos apontam que o principal motivo de encaminhamentos para eles ocorre devido a condições crônicas prevalentes mal controladas na APS, sendo considerados pelos endocrinologistas como mal indicados. O médico regulador avalia que a maioria dos documentos de encaminhamento é incompleta e não permite verificar a gravidade do problema. Conclui-se que os protocolos de encaminhamento não estão sendo utilizados de forma a otimizar os fluxos dos usuários na rede de atenção à saúde, indicando a necessidade de revisão de processos de trabalho, capacitação dos profissionais e articulação entre APS, regulação e Atenção Especializada.

PALAVRAS-CHAVE Doenças crônicas. Endocrinologia. Cardiologia. Atenção Primária à Saúde. Serviços de saúde.

\begin{abstract}
This study aimed to analyze referrals of people to cardiology and endocrinology services by doctors in Primary Health Care (PHC) in a city in Rio Grande do Sul. Cross-sectional study with questionnaires applied to $25 \mathrm{PHC}$ doctors, 2 cardiologists, 2 endocrinologists and 1 regulatory doctor. Statistical analysis was performed in SPSS, with continuous variables being presented as mean or median; and categorical variables, such as absolute and relative frequencies. Among PHC doctors, 96\% know, 84\% use referral protocols, and 92\% refer patients mainly to manage complicated diseases that require specialist evaluation. Among the specialists, 50\% know the protocols, and all point out that the main reason for referrals to them occurs due to prevalent chronic conditions poorly controlled in $\mathrm{PHC}$, being considered by endocrinologists as poorly indicated. The regulatory doctor assesses that most referral documents is incomplete and does not allow to verify the seriousness of the problem. It is concluded that the referral protocols are not being used in order to optimize the flow of users in the health care network, indicating the need for review of work processes, training of professionals and articulation between $P H C$, regulation and Specialized Care.
\end{abstract}

KEYWORDS Chronic disease. Endocrinology. Cardiology. Primary Health Care. Health services. 


\section{Introdução}

As Doenças Crônicas Não Transmissíveis (DCNT) constituem atualmente as principais causas de morte no mundo, tendo como característica o início progressivo, o prognóstico normalmente incerto e a duração longa ou indefinida. Nos seus trajetos, possuem períodos críticos que podem levar à incapacitação, possuindo, assim, a necessidade de uma atenção contínua ${ }^{1}$. Na esfera global, segundo a Organização Mundial da Saúde, as DCNT foram responsáveis, em 2015, por cerca de 40 milhões de mortes, o que representa, aproximadamente, $70 \%$ do total da mortalidade ocorrida naquele ano ${ }^{2}$.

As Doenças Cardiovasculares (DCV) são, entre as DCNT, as mais prevalentes e com os maiores coeficientes de morbimortalidade, especialmente quando associadas ao Diabetes Mellitus tipo 2 (DM2). No Brasil, em 2015, as DCV geraram 807.304 internações clínicas e 279.010 internações cirúrgicas; e o custo provocado por essas patologias e suas consequências foi de cerca de $\mathrm{R} \$ 37$ bilhões, representando 0,7\% do Produto Interno Bruto (PIB) nacional $^{3}$. Da mesma forma, segundo a Federação Internacional de Diabetes, em 2017, o Brasil esteve na quarta posição mundial em relação ao número de pessoas com DM2 entre os 20 anos e 79 anos, com o acometimento de cerca de 12,5 milhões de pessoas, sendo 5,7 milhões sem diagnóstico ${ }^{4}$, gerando um custo para o sistema de saúde estimado, em 2015, de cerca de 24 US $\$$ bilhões ${ }^{4}$. No estado do Rio Grande do Sul (RS), essas doenças também possuem uma grande relevância: o coeficiente de mortalidade por DCV aumentou de 127 em 2013 para 155,7/100 mil hab. em 2016; e por DM2, o coeficiente de mortalidade passou de 39,6 para 53,2/100 mil hab. no mesmo período 5 .

No Brasil, os serviços públicos de saúde são promovidos pelo Sistema Único de Saúde (SUS), que possui como diretrizes e princípios: universalidade, equidade, integralidade, direito à informação, resolutividade, autonomia das pessoas e epidemiologia como base.
No entanto, um dos grandes problemas do SUS é o acesso à consulta especializada, inclusive para o manejo das DCNT ${ }^{6}$. Muitos usuários com DCV e DM2 necessitam de avaliação em serviço ambulatorial especializado, seja para a realização de exames complementares não disponíveis na Atenção Primária à Saúde (APS), seja para melhor avaliação diagnóstica ou otimização de um tratamento que já está sendo oferecido. A dificuldade e o tempo prolongado de espera para o acesso aos serviços ambulatoriais especializados decorrem de fatores como o modelo de atenção adotado, o dimensionamento e a organização das ofertas, bem como do grau de resolutividade local da APS. Esses gargalos requerem, para a sua superação, que se constituam estratégias que impactem na APS, nos processos de regulação do acesso (desde os serviços solicitantes até as centrais de regulação), bem como na organização da Atenção Especializada (AE) ${ }^{7,8}$.

$\mathrm{O}$ uso dos protocolos de encaminhamento da APS para a AE, disponibilizados, desde 2013, pelo Projeto TelessaúdeRS em parceria com a Universidade Federal do Rio Grande do Sul e a Secretaria de Saúde do RS e incorporados pelo Ministério da Saúde (MS), otimiza a regulação das referências, orienta a priorização do atendimento para os pacientes mais graves e atenta os médicos da APS para as condições que podem ser resolvidas em seu próprio município e aquelas que devem ser encaminhadas para a AE. O protocolo de endocrinologia foi um dos primeiros a ser elaborado em 2013 e foi publicado em Resolução CIB/RS 171/2013; enquanto o protocolo de cardiologia foi elaborado em 2014 e publicado conforme Resolução CIB/ $\mathrm{RS} \mathrm{n}^{0} 370 / 14$. Ambos foram revisados conforme resolução CIB/RS 764/20149,10. Tanto o RS como o MS disponibilizam e recomendam o uso desses documentos para os gestores e os profissionais de saúde que atuam nos serviços públicos de APS como guias para o encaminhamento mais preciso de várias condições clínicas de diferentes especialidades médicas.

Quando os usuários são encaminhados aos serviços especializados de cardiologia e 
endocrinologia sem indicação, formam-se filas de espera e aumenta a demanda reprimida ao $\mathrm{AE}$. Essa situação dificulta o acesso dos usuários que realmente precisam do manejo dos profissionais especialistas, o que prejudica o fluxo na Rede de Atenção à Saúde (RAS). $\mathrm{O}$ acesso à $\mathrm{AE}$ requer articulação regional para que ocorra de forma regulada, equitativa e oportuna, evitando-se a demanda reprimida e adotando-se protocolos pactuados no sistema locorregional. Esses protocolos ordenam o acesso e minimizam a realização de múltiplos exames diagnósticos sem adequada indicação clínica11.

Não se encontraram estudos que tenham avaliado o uso dos protocolos de encaminhamento pelos profissionais da APS em municípios pequenos, com regulação local; assim como estudos que tenham avaliado a adequação dos encaminhamentos da APS pelos médicos reguladores ou pelos médicos da AE. Dessa forma, o objetivo deste estudo foi analisar os processos de encaminhamento de pessoas com DCV e DM2 a serviços de cardiologia e endocrinologia pelos médicos da APS.

\section{Material e métodos}

Realizou-se um estudo quantitativo, observacional, do tipo transversal, por meio da aplicação de questionários, elaborados e aplicados pelo pesquisador, aos médicos da APS, ao médico regulador e aos médicos dos serviços de endocrinologia e cardiologia de um município de médio porte, no interior do RS. A RAS do município conta com 14 equipes de Estratégia Saúde da Família (ESF), 3 Centros de Saúde (CS), 1 Unidade Básica de Saúde (UBS), 1 Unidade de Pronto Atendimento (UPA), 1 Hospital macrorregional e um Ambulatório de Especialidades Médicas Universitário (Aemu).

$O$ acesso da população aos serviços de cardiologia e endocrinologia do Aemu, pelo SUS, se dá por meio do primeiro contato com os profissionais médicos da APS, que avaliam a necessidade de encaminhamento para a AE. $\mathrm{O}$ documento de referência para a $\mathrm{AE}$ é avaliado pelo médico regulador, que verifica sua adequação e determina a prioridade da consulta.

Todos os médicos generalistas dos serviços de APS do município ( $\mathrm{n}=27$ ), o médico regulador ( $\mathrm{n}=1)$, os médicos cardiologistas $(\mathrm{n}=2)$ e endocrinologistas $(n=2)$ do Aemu foram convidados a participar do estudo. Estes eram os profissionais que atuavam no período da coleta, que ocorreu entre o dia $1^{\circ}$ de agosto e 15 de novembro de 2018.

Três questionários diferentes foram aplicados, um para os médicos da APS (19 perguntas), um para o médico regulador (21 perguntas) e um para os médicos da AE (18 perguntas). Dados pessoais, de formação e de processo de trabalho foram itens comuns aos três questionários: idade, local de nascimento, sexo, ano de graduação, nome da instituição e local da instituição de graduação, especialidade médica, maior título acadêmico, tempo de trabalho nos serviços do SUS de APS, regulação e AE e tempo de trabalho no serviço na RAS do município, conhecimento dos protocolos de encaminhamento do estado/MS (sim/não) e utilização desses protocolos como base para o encaminhamento (sim/não).

O questionário dos médicos da APS incluiu perguntas sobre os motivos para que os protocolos não sejam utilizados (não conheço esses materiais; não estão facilmente disponíveis; não são práticos para uso diário; não concordo com os critérios utilizados; não é uma exigência do município onde atuo; outro, qual?) e as situações que com maior frequência geram o encaminhamento de pacientes da APS para a AE (para solicitação de exames não disponíveis na atenção primária; para avaliação e tratamento de condições cujo manejo não domina; para manejo de doenças complicadas que necessitam de avaliação do especialista, independentemente dos protocolos de encaminhamento disponíveis; para investigação ou manejo de doenças, após o manejo possível na atenção básica, seguindo as recomendações dos protocolos disponíveis; por insistência do paciente ou da família; a pedido de outros médicos que avaliaram 
o paciente anteriormente; outros, qual? podendo ser assinalada mais de uma opção). Nessa última pergunta, mais de uma opção poderia ser assinalada. $\mathrm{O}$ uso dos canais do TelessaúdeRS para discussão de casos clínicos ou regulação de encaminhamentos também foi questionado ( $\operatorname{sim} /$ não).

O questionário para o médico regulador abordou: a experiência prévia de trabalho na APS; o uso dos protocolos de encaminhamento no processo de regulação e os motivos para não os utilizar (não estão facilmente disponíveis; não são práticos para o uso diário; não concordo com os critérios utilizados; não é uma exigência do município onde atuo; utilizo protocolos regionais ou locais, quais? Utilizo outros critérios para a regulação dos encaminhamentos; outro motivo, qual?); a regulação em si (autorização e priorização, cancelamento, devolução à APS por pendências); a qualidade dos encaminhamentos (bons: completos, permitem avaliar gravidade do problema; regulares: incompletos, não permitem avaliar gravidade do problema; ruins: não especificam o motivo do encaminhamento nem a sua gravidade); e o uso do canal do TelessaúdeRS para regulação de casos e se há estímulo para que os médicos da APS o utilizem (sim/não).

O questionário para os médicos cardiologistas e endocrinologistas da AE incluiu perguntas sobre sua percepção da utilização dos protocolos como base para os encaminhamentos que recebem; os motivos de encaminhamento que com maior frequência chegam ao seu ambulatório (pacientes encaminhados para solicitação de exames não disponíveis na atenção primária; pacientes encaminhados por condições prevalentes mal controladas na atenção primária; pacientes encaminhados para manejo de doenças graves, complicadas ou raras que necessitam avaliação do especialista; pacientes encaminhados para investigação ou manejo de doenças, após manejo possível na atenção primária, seguindo a recomendação de protocolos disponíveis; pacientes encaminhados por insistência do paciente ou de sua família; pacientes encaminhados pelos médicos da atenção primária por solicitação de outros especialistas; outros, qual? - podendo ser assinalada mais de uma opção); a qualidade dos encaminhamentos (bons: encaminhamentos bem feitos, bem indicados; ruins: encaminhamentos mal feitos, mal indicados); se há solicitação prévia para que os protocolos sejam utilizados como base para o encaminhamento a sua especialidade e se há contato com médicos da APS para discussão de casos clínicos atendidos no ambulatório (sim/não).

Após a coleta dos dados, a análise estatística foi realizada no programa Statistical Package for the Social Sciences $\left(\right.$ SPSS $^{\circledR}$ ) versão 25.0. As informações foram apresentadas da seguinte forma: as variáveis contínuas, como médias \pm desvio-padrão ou mediana (intervalo interquartil); e as variáveis categóricas, como frequência absoluta (número) e relativa (porcentagem).

O projeto foi aprovado pelo Comitê de Ética em Pesquisa (Coep) da Universidade do Vale do Taquari (Univates), sob o número 2.196.011; e todos os participantes assinaram o Termo de Consentimento Livre e Esclarecido (TCLE).

\section{Resultados}

\section{Perfil sociodemográfico dos médicos da atenção primária e especializada}

O perfil dos profissionais da APS e da AE está descrito na tabela 1. Dos 27 médicos da APS convidados, 25 aceitaram participar do estudo (um não aceitou e o outro não pôde participar por fazer parte do grupo de pesquisadores). Quatro médicos que trabalham no Aemu, sendo 2 cardiologistas e 2 endocrinologistas, e o médico regulador que trabalha na Secretaria Municipal da Saúde (Sesa) responderam aos questionários da pesquisa.

A média de idade dos médicos da APS é de $38,2 \pm 11,9$ anos, sendo a idade mínima de 26 anos, e a máxima, de 75 anos; a média do tempo de formação de 12,3 $\pm 11,5$ anos, tendo o profissional com a formação mais antiga concluída no ano de 
1976, e o mais novo, no ano de 2018; a mediana de tempo de trabalho na APS é de $60(12$ - 172) meses; e a mediana de tempo de trabalho na APS do município é de $17(5-48)$ meses. Oito profissionais da APS são cubanos; destes, sete possuem, além da formação, especialização em medicina generalista, e um, com especialização em medicina de família; enquanto os profissionais brasileiros $(n=17)$ possuem uma formação heterogênea, em várias especialidades. Apenas uma médica brasileira tem formação generalista, que foi concluída em Cuba.
Com relação aos quatro médicos da $\mathrm{AE}$, estes possuem: média de idade de $47,3 \pm 5$ anos, sendo a idade mínima de 40 , e a máxima, de 51; a média do tempo de formação de $23,3 \pm 6,6$ anos, tendo o profissional com a formação mais antiga concluída em 1991, e com a mais nova, em 2005; a mediana do tempo de trabalho no SUS é de $30(23,5$ - 133,5) meses; e a média do tempo de trabalho no Aemu de 23,6 $\pm 5,1$ meses, em que o mínimo foi de 18 meses, e o máximo, de 30 meses ( 2 anos e meio).

Tabela 1. Perfil sociodemográfico dos médicos da atenção primária e Atenção Especializada

\begin{tabular}{|c|c|c|c|c|}
\hline & \multicolumn{2}{|c|}{ Atenção Primária $(\mathrm{n}=25)$} & \multicolumn{2}{|c|}{ Atenção Especializada $(n=4)$} \\
\hline & $\mathrm{n}$ & $\%$ & $\mathbf{n}$ & $\%$ \\
\hline \multicolumn{5}{|l|}{ Sexo } \\
\hline Masculino & 6 & 24 & 2 & 50 \\
\hline Feminino & 19 & 76 & 2 & 50 \\
\hline \multicolumn{5}{|l|}{ Idade } \\
\hline De 26 a 36 anos & 15 & 60 & - & - \\
\hline De 37 a 47 anos & 5 & 20 & 1 & 25 \\
\hline De 48 a 58 anos & 3 & 12 & 3 & 75 \\
\hline Acima de 59 anos & 2 & 8 & - & - \\
\hline \multicolumn{5}{|l|}{ Nacionalidade } \\
\hline Brasileira & 17 & 68 & 4 & 100 \\
\hline Cubana & 8 & 32 & - & - \\
\hline \multicolumn{5}{|c|}{ Tempo de conclusão da graduação } \\
\hline Menos de 10 anos & 13 & 52 & - & - \\
\hline De 10 a 20 anos & 6 & 24 & 1 & 25 \\
\hline Há mais de 20 anos & 6 & 24 & 3 & 75 \\
\hline \multicolumn{5}{|c|}{ Maior título acadêmico } \\
\hline Graduação & 4 & 16 & - & - \\
\hline Pós-graduação & 12 & 48 & - & - \\
\hline Residência médica & 7 & 28 & - & - \\
\hline Mestrado & 2 & 8 & 3 & 75 \\
\hline Doutorado & - & - & 1 & 25 \\
\hline \multicolumn{5}{|l|}{ Especialidade ${ }^{\star}$} \\
\hline Cardiologia & - & - & 2 & 50 \\
\hline Clínica médica & 10 & 47,6 & - & - \\
\hline Cirurgia geral & 2 & 9,5 & - & - \\
\hline Dermatologia & 1 & 4,8 & - & - \\
\hline
\end{tabular}


Tabela 1. (cont.)

\begin{tabular}{lcccc}
\hline & Atenção Primária $(\mathbf{n}=\mathbf{2 5})$ & Atenção Especializada $(\mathbf{n}=\mathbf{4})$ \\
& $\mathbf{n}$ & $\mathbf{\%}$ & $\mathbf{n}$ & \% \\
\hline Endocrinologia & - & + & 2 & 50 \\
Geriatra & 1 & 4,8 & - & - \\
Ginecologia/Obstetrícia & 1 & 4,8 & - & - \\
Medicina do Trabalho & 2 & 9,5 & - & - \\
Medicina de Família & 2 & 9,5 & - & - \\
Nefrologia & 1 & 4,8 & - & - \\
Psiquiatria & 1 & 4,8 & - & - \\
\hline
\end{tabular}

Fonte: Elaboração própria.

*Os especialistas apresentaram um $n=21$

\section{Resultados dos questionários aplicados aos médicos da atenção primária}

Com relação aos protocolos de encaminhamento para a AE, $96 \%(n=24)$ dos médicos responderam que conhecem, e $84 \%(n=21)$ responderam que os utilizam em sua rotina. Os médicos que relataram que não utilizam alegaram que: 1) seu uso não é exigência do município no qual atua $(\mathrm{n}=1) ; 2$ ) os documentos não estão facilmente disponíveis (n=1); 3) não concorda com os critérios utilizados (n=1); e 4) na maioria dos casos, independentemente dos protocolos, necessita encaminhar para o especialista pela necessidade de solicitação de exames complementares $(n=1)$.

Com relação à utilização do TelessaúdeRS para auxílio na discussão de casos e na regulação de encaminhamentos para a AE, 96\% $(\mathrm{n}=24)$ dos médicos da APS relataram que utilizam esse serviço. Os motivos, apontados pelos médicos, que os levam a encaminhar os pacientes para AE são mostrados no gráfico 1.

Gráfico 1. Motivos de encaminhamentos apontados pelos médicos da atenção primária

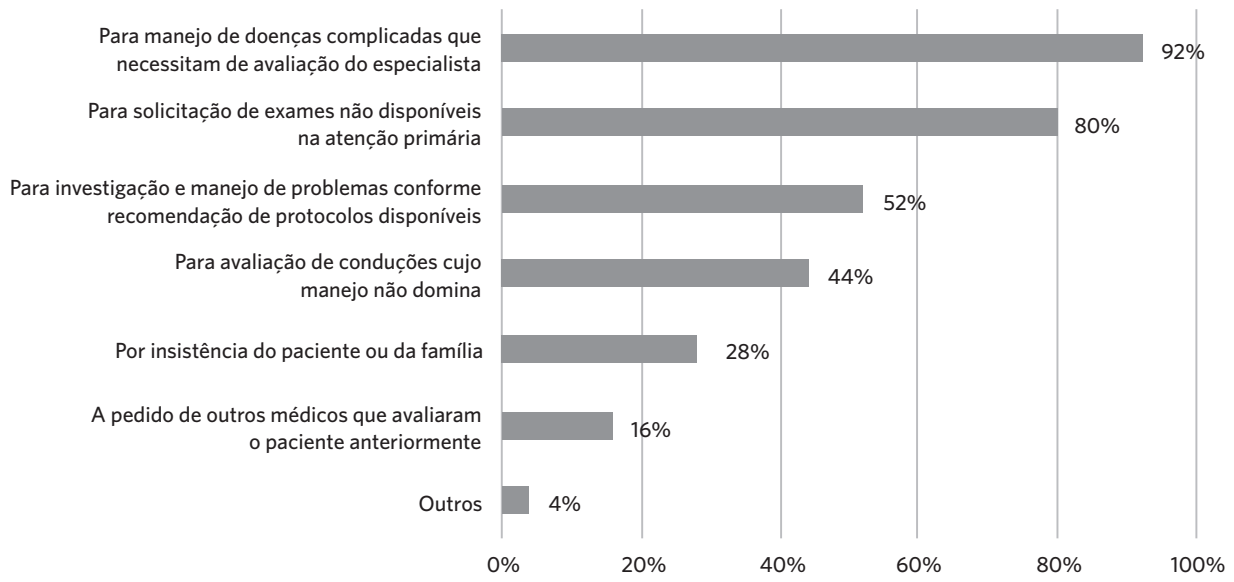

Fonte: Elaboração própria. 


\section{Resultados do questionário aplicado ao médico regulador}

O médico regulador tem por função avaliar os encaminhamentos realizados por todos os médicos da APS, com o objetivo de verificar sua pertinência, considerando protocolos de encaminhamento para a atenção secundária.

A regulação dos pacientes da APS para a AE do município é realizada apenas por um profissional - uma médica, de 31 anos, brasileira, natural do RS, que concluiu sua graduação em 2016, sendo esse seu maior título acadêmico. Trabalha na regulação há um ano e tem experiência de um ano de trabalho na APS do próprio município.

Conhece os protocolos de encaminhamento para especialidades médicas do Estado e do MS e os utiliza durante a regulação. Tem a prerrogativa de autorizar ou cancelar os encaminhamentos para a $\mathrm{AE}$, conforme a adequação da referência feita pela APS, devolvendo-os para esta, quando não estiverem de acordo com os critérios previamente estabelecidos. Avalia, na média, que a qualidade dos encaminhamentos é regular (incompletos, que não permitem avaliar a gravidade do problema).

Além de utilizar os recursos do TelessaúdeRS para a regulação, estimula os médicos da APS a utilizarem-no para auxílio na discussão de casos (canal 0800) e para o encaminhamento correto de pacientes (protocolos).

\section{Resultados do questionário aplicado aos médicos do serviço de Atenção Especializada de cardiologia e endocrinologia}

Entre os médicos da AE, $50 \%(\mathrm{n}=2)$ responderam que conhecem os protocolos de encaminhamento, sendo 1 cardiologista e 1 endocrinologista; e 50\% $(n=2)$ responderam que os desconheciam, sendo, também, 1 cardiologista e 1 endocrinologista.

Sobre a avaliação da qualidade dos encaminhamentos que esses médicos recebem no Aemu, os cardiologistas responderam que são adequados (bem feitos, bem indicados); e os endocrinologistas responderam que são ruins (mal feitos, mal indicados).

Também com relação ao uso de protocolos, $75 \%(n=3)$ dos médicos do Aemu afirmam que já solicitaram à regulação que esses documentos fossem utilizados como base para os encaminhamentos, de forma a otimizar o acesso aos pacientes (a resposta negativa foi de um endocrinologista). Da mesma forma, $75 \%(n=3)$ afirmam que já entraram em contato com os médicos da APS para discutir caso de paciente encaminhado (sendo a resposta negativa de um endocrinologista).

O gráfico 2 mostra os motivos mais comuns dos encaminhamentos que chegam ao Aemu, sob o ponto de vista dos cardiologistas e endocrinologistas.

Gráfico 2. Motivos de consultas apontados pelos médicos da Atenção Especializada de endocrinologia e cardiologia

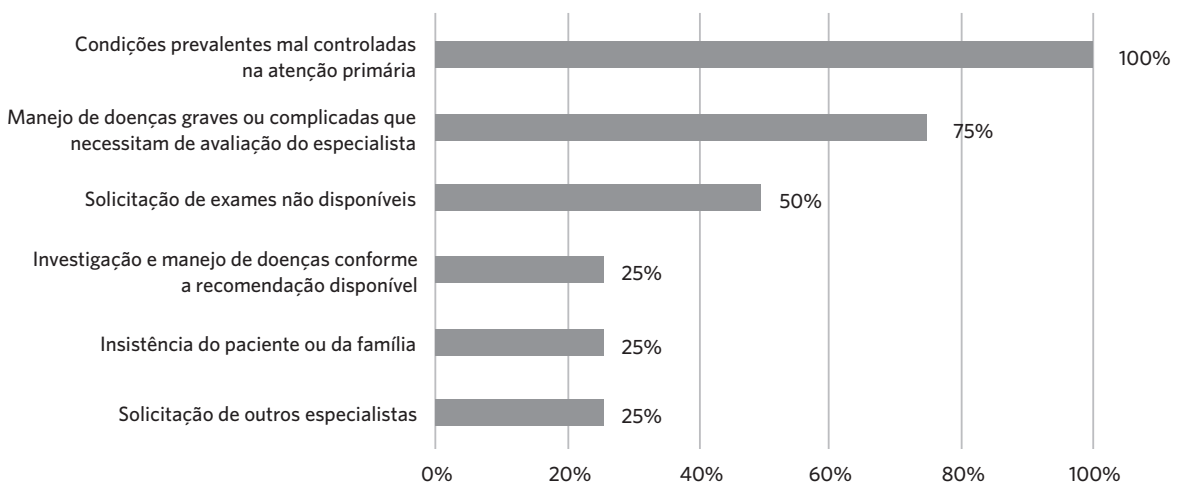




\section{Discussão}

As respostas dos médicos da APS revelaram o conhecimento e o uso dos protocolos de encaminhamentos e o contato destes com os profissionais do TelessaúdeRS para discussão de casos, demonstrando que eles estão cientes da demanda reprimida, dos mecanismos disponibilizados pelos gestores para a sua adequação, e se dispõem a aumentar sua resolutividade com o suporte do TelessaúdeRS. O médico regulador e os médicos da Aemu também estão cientes e utilizam esses protocolos na sua rotina de trabalho. A regulação assistencial por meio de protocolos permite um filtro dos encaminhamentos desnecessários, em que serão selecionados apenas os pacientes que possuem indicação clínica a alguma consulta e/ou procedimento, o que gera maior expansão do cuidado e resolutividade pela APS, como também o melhor uso de recursos em saúde, maior eficiência assistencial e igualdade/ equidade ao acesso em saúde ${ }^{7,8}$. No entanto, estudos mostram que as centrais de regulação podem operar restringindo a oferta a tetos pré-acordados ${ }^{12}$. Embora os profissionais da APS conheçam e utilizem esses protocolos, sob o ponto de vista do médico regulador, os documentos de referência ainda carecem de adequação. Além disso, apesar de o município dispor de um médico regulador, que deveria filtrar encaminhamentos inadequados, na opinião dos endocrinologistas, a qualidade dos encaminhamentos que chegam a esse ambulatório é, em sua maioria, ruim. Para os médicos da Aemu, a maioria dos encaminhamentos ocorre por condições prevalentes mal controladas na APS; já para os médicos da APS, esses encaminhamentos ocorrem por condições complicadas que necessitam de avaliação de especialista e para a realização de exames diagnósticos. Esses achados demonstram incongruência nas informações e nas percepções dos profissionais dos diferentes serviços e uma problemática das RAS, tanto estrutural como de comunicação e treinamento, o que aumenta a fila de espera para consulta com especialistas e onera os cofres públicos com encaminhamentos desnecessários. Dessa forma, o ideal seria que a decisão de acesso fosse tomada de forma interativa entre o especialista e o profissional de referência ${ }^{12}$.

Segundo a Política Nacional de Atenção Básica (PNAB), são responsabilidades comuns a todas as esferas de governo desenvolver mecanismos técnicos e estratégias organizacionais de qualificação da força de trabalho para gestão e atenção à saúde, estimular e viabilizar a formação, educação permanente e continuada dos profissionais, visando à qualificação dos serviços ofertados às pessoas ${ }^{6}$. Por sua vez, compete às secretarias municipais da saúde garantir acesso ao apoio diagnóstico e laboratorial necessário ao cuidado resolutivo da população ${ }^{6}$. Tanto a educação permanente e continuada para qualificação dos profissionais e, especificamente, do processo de encaminhamento como o aporte de recursos que permitam maior resolutividade à APS são intervenções apropriadas para o município. Conforme Starfield ${ }^{\mathbf{1 3}}$, os médicos da APS estão mais próximos do ambiente do paciente do que os especialistas, sendo capazes de avaliar melhor o papel dos múltiplos e interativos determinantes da doença e da saúde13. São eles que exploram a natureza do problema e iniciam o trabalho de diagnóstico preliminar13. À AE, cabe o processo diagnóstico com definição precisa da fisiopatologia e intervenções orientadas para esse processo fisiopatológico específico13. É a APS, portanto, que forma a base e determina o trabalho dos demais níveis do sistema de saúde, organizando e racionalizando o uso dos recursos, tanto básicos como especializados, direcionados para a promoção, manutenção e melhora da saúde. Com os recursos adequados, a APS pode cumprir suas funções primordiais, tais como a resolutividade de $85 \%$ das patologias mais comuns em saúde; a função de protagonista na coordenação (comunicação) dos fluxos de referência e contrarreferência de pessoas, de produtos e de informações; e, também, de responsabilização da equipe de saúde em relação aos usuários da RAS ${ }^{\mathbf{1 4}}$. 
Além da disponibilização de protocolos de encaminhamento, é fundamental que a gestão da saúde do município estimule e disponibilize outros recursos para os serviços da APS, como teleconsultorias de maneira integrada entre a APS, o centro de regulação e a $\mathrm{AE}$, para que o apoio assistencial possa reduzir filas de espera e aumentar o acesso dos pacientes que realmente necessitam de consultas e procedimentos especializados, o que promove o atendimento de qualidade na APS e evita deslocamentos e custos desnecessários ${ }^{15,16}$. O Telessaúde é fundamental no apoio aos profissionais que atuam na APS, pois oferece teleconsultorias, telediagnóstico, segunda opinião formativa e tele-educação, que ajudam a superar as dificuldades vivenciadas no manejo clínico e de gestão nos serviços de APS ${ }^{17}$. Segundo estudos realizados nos estados de Minas Gerais e do RS, a teleconsultoria foi capaz de reduzir, aproximadamente, $70 \%$ dos encaminhamentos, sendo esses casos resolvidos na própria UBS ${ }^{18}$.

O papel do regulador é de extrema importância na coordenação/ordenação dos fluxos de pacientes entre APS e as outras estruturas da RAS, visto que, para a resolução da problemática assistencial, principalmente no que se refere ao acesso ao serviço especializado, torna-se fundamental a criação de mecanismos que superem essa dificuldade estrutural, com estratégias que melhorem a APS, a regulação do acesso do usuário, bem como a organização da $A E^{7,8}$. Uma RAS desorganizada torna inadequada a assistência ofertada, gerando insatisfação, atraso no tratamento e perda de recursos. Por isso, o trabalho do regulador exige conhecimento técnico e de gestão, para ter a capacidade de observar qual é a melhor assistência que o sistema de saúde pode ofertar de acordo com as necessidades apresentadas. É importante que esse profissional aponte as carências da RAS ao gestor, para auxiliá-lo na priorização dos investimentos nas várias áreas ${ }^{19}$.

A reguladora do município do estudo, embora tenha pouco tempo de experiência e não tenha formação específica na área de gestão, parece seguir esses preceitos, já que conhece, estimula o uso e utiliza os protocolos de encaminhamento no seu processo de trabalho de regulação, e pode questionar, devolver para APS ou cancelar referências inadequadas. Apesar disso, os encaminhamentos que chegam à especialidade de endocrinologia foram classificados como ruins. O processo de regulação em si, o uso de outras ferramentas durante o processo e a classificação de risco, entretanto, não foram avaliados neste estudo.

Nessa mesma perspectiva de otimização dos encaminhamentos, o projeto RegulaSUS, sistema que utiliza os protocolos e possibilita a discussão de casos clínicos entre médicos da APS e médicos teleconsultores e telerreguladores da equipe do TelessaúdeRS, evitou, por telefone, $60 \%$ dos encaminhamentos da APS para outros serviços da RAS ${ }^{20}$. Dados do estudo revelam que houve redução da fila de espera do serviço especializado de endocrinologia em $52 \%$; de nefrologia, em $38 \%$; de pneumologia, em $59 \%$; de neurologia, em $25 \%$; e de estomatologia, em $71 \%$, no período de um ano (entre fevereiro de 2015 e fevereiro de 2016) ${ }^{\mathbf{2 0}}$. Dessa forma, o uso dos protocolos de encaminhamento, aliado à regulação correta das condições clínicas referenciadas, constitui um mecanismo primordial para evitar os deslocamentos desnecessários, diminuir o tempo de espera dos pacientes e permitir acesso a quem realmente precisa da consulta especializada.

O sistema de saúde de Portugal organiza o acesso aos serviços de saúde de forma semelhante ao SUS no Brasil: critérios para a referência de pacientes, com diferentes condições clínicas, aos demais níveis de atenção estão disponíveis nas Normas da DireçãoGeral de Saúde - linhas de cuidado elaboradas pelos colégios de diversas especialidades em conjunto com a sociedade de medicina de família e comunidade ${ }^{21,22}$. As linhas de cuidado para pacientes com DM2 e DCV, por exemplo, mostram as atribuições da APS, os critérios de encaminhamento para as subespecialidades e disponibilizam a organização dos fluxos até a rede hospitalar ${ }^{21,22}$. O processo 
de referenciamento dos pacientes é informatizado, e há médicos responsáveis para avaliar e dar a prioridade adequada aos encaminhamentos realizados pela APS - o que se assemelha à nossa regulação ${ }^{21,22}$. Uma diferença importante da assistência entre os dois países é que, em Portugal, o acesso a exames complementares está disponível aos serviços de APS, alguns deles também com critérios bem definidos para a sua solicitação, não havendo, como em alguns serviços de saúde no Brasil, a necessidade de encaminhamento única e exclusivamente para solicitação de exames diagnósticos complementares $\mathbf{2 1 , 2 2}^{2}$.

No Canadá, a rede de atenção primária também é a base do sistema de saúde. O médico de família é o profissional responsável pelo seguimento horizontal e encaminha para especialistas seguindo a característica de racionalidade aceita em quase todos os sistemas de saúde universais do mundo. Praticamente não há um modelo privado de saúde nos moldes como conhecemos no Brasil, e a ideia é de que a capacidade de compra do cidadão possa lhe permitir maior acesso à estrutura de saúde é repudiada pela maioria dos canadenses ${ }^{23}$.

Sob o ponto de vista dos profissionais da Aemu, as condições prevalentes mal controladas na APS consistem no principal motivo dos encaminhamentos. Essa perspectiva demonstra a falha da APS no manejo de condições clínicas comuns, como Hipertensão Arterial Sistêmica (HAS) e DM2 - DCNT que são primordialmente de sua atribuição, o que reflete um grande número de encaminhamentos realizados sem o cumprimento dos critérios fornecidos pelos protocolos do MS. O encaminhamento correto de HAS deve ocorrer somente nas condições clínicas de suspeita de hipertensão secundária ou por motivo de hipertensão mal controlada, mesmo com a adesão correta do paciente ao uso de, no mínimo, três drogas anti-hipertensivas em dose plena; além de ter descrito: sinais e sintomas, medicações em uso com posologia, duas medidas de pressão arterial em dias diferentes, alterações em exames, avaliação clínica da adesão ou não ao tratamento e o número de teleconsultoria, se caso foi discutido com o Telessaúde ${ }^{8}$. Já nos casos de DM2, as condições clínicas que necessitam de encaminhamento são: paciente em uso de insulina com mais de uma unidade por quilograma do peso ou com doença renal crônica, tendo Taxa de Filtração Glomerular (TFG) < $30 \mathrm{ml} / \mathrm{min} / 1,73 \mathrm{~m}^{2}$ ou paciente portador de Diabetes Mellitus Tipo 1 em uso de insulina como medicação principal; além do conteúdo descritivo: resultado de hemoglobina glicada e creatinina sérica, ambos com data, se utiliza ou não insulina com dose e posologia, outras medicações em uso com dose e posologia, peso em quilogramas $(\mathrm{kg})$ do paciente e o número da teleconsultoria, se o caso foi discutido com o Telessaúde? ${ }^{7}$. Apesar da disponibilidade desses critérios de encaminhamento nos protocolos, os documentos de referência ainda não trazem as informações devidas, pois o próprio regulador do município os considera incompletos.

Os dois profissionais endocrinologistas avaliaram os encaminhamentos para a especialidade como ruins, em sua maioria. Estudo prévio demonstrou que o controle de pacientes com DM2 nos serviços públicos de saúde no Brasil está aquém das metas estabelecidas ${ }^{24}$. A dificuldade em atingir as metas, assim como a falta de familiaridade com o manejo das insulinas, pode contribuir para o encaminhamento precoce e mal indicado de pacientes para $\mathrm{AE}$, antes mesmo que todas as medidas farmacológicas e não farmacológicas sejam oferecidas e seguidas pelos pacientes.

Com relação às DCV, a necessidade de encaminhamento para manejo clínico ou avaliação diagnóstica pode ser avaliada pelos cardiologistas, em sua maioria, como adequada, na medida em que, no município, o acesso a exames complementares na APS se restringe ao eletrocardiograma em repouso. A AE, fundamental na RAS, representa um apoio à APS nos casos de maior complexidade, a fim de evitar complicações de morbidades, por meio da disponibilização dos médicos especialistas para a resolução de casos complicados, de 
recursos tecnológicos para ajudar no diagnóstico e da garantia do acesso a medicamentos ${ }^{25}$. Como característica ideal, as RAS precisam levar em consideração a equidade, a integralidade e a eficácia, entre os serviços da APS e $\mathrm{AE}$, para que se tenha a garantia das referências adequadas e um mecanismo de triagem apropriado sem barreiras no acesso, assim como a contrarreferência assegurada após o fim da assistência ao usuário na $\mathrm{AE}^{\mathbf{2 6}}$.

Este estudo apresentou limitações na busca de referências sobre a avaliação da utilização de protocolos de encaminhamento, da gestão dos encaminhamentos e na análise dos seguintes aspectos: 1) se o uso dos protocolos consegue ou não evitar o encaminhamento dos pacientes em situações desnecessárias, visto que a maioria dos encaminhamentos ocorre pela falta de recursos ou treinamento da APS, e não apenas para o melhor manejo clínico de situações complicadas como deveria ser; 2 ) em quais situações ou quais são os critérios utilizados pelo médico regulador no processo de regulação, além do uso do protocolos disponíveis; 3) se a formação acadêmica, a nacionalidade, o tempo da graduação e o tempo de serviço na APS interferem diretamente nos motivos e qualidade dos encaminhamentos.

A maioria dos médicos da APS são mais jovens, formados há menos tempo e têm menor título acadêmico em comparação com os médicos da AE. Outro aspecto observado é que, na APS, os médicos - especialmente os brasileiros - têm os mais variados tipos de especialidades, e o tempo de trabalho na APS apresenta um grande desvio-padrão, com médicos que trabalham há 40 anos, e outros, há apenas um mês, demonstrando um grupo muito heterogêneo, em que a maioria não direcionou ou não está direcionando sua formação para a APS. Dessa forma, o crescimento da APS não veio acompanhado do suficiente aumento na quantidade de médicos formados, muito menos no número de médicos de família, mesmo com o programa Mais Médicos ${ }^{27}$. Esse fato provavelmente leva à rotatividade de profissionais, com a consequente ausência de vínculos de longo prazo com a equipe e a população, comprometendo a dinâmica da APS, visto que os médicos acabam por não conhecer a população da região e suas principais mazelas, bem como não se adéquam por completo aos processos de trabalho, tais como os de encaminhamentos aos demais níveis de atenção. Para minimizar esse problema, é necessário o incentivo à formação em medicina de família e comunidade. Esta é reconhecida internacionalmente como a especialidade médica de excelência da APS, sendo estratégica para o seu pleno desenvolvimento, e é considerada a especialidade com potencial estruturante para os sistemas nacionais de saúde ${ }^{\mathbf{2 8}}$.

Os profissionais com especialidades diversas daquelas relacionadas com a APS eram todos brasileiros, e os médicos de nacionalidade cubana tinham formação em medicina generalista ou medicina de família. O presente estudo não avaliou diferenças entre os encaminhamentos dos profissionais de diferentes nacionalidades ou se uma formação não generalista pode contribuir para encaminhamentos desnecessários. Por outro lado, diferenças socioculturais, de epidemiologia e prevalência de doenças e de funcionamento do sistema da saúde podem também dificultar o entendimento e a utilização correta das RAS e de protocolos. Mais estudos são necessários para avaliar se esses fatores influenciam, de fato, os encaminhamentos da APS para a AE.

\section{Conclusões}

O estudo demonstrou que a maioria dos médicos da APS refere utilizar os protocolos disponíveis para encaminharem os pacientes da APS para a AE. Mesmo assim, o processo de encaminhamento ainda necessita de adequação, pois os protocolos não são capazes de sanar a problemática da APS no que tange à resolutividade, uma vez que os médicos da APS têm sua ação limitada pela falta de recursos diagnósticos.

Para melhorar o processo de encaminhamento e regulação, a gestão do município 
precisa implementar mudanças no processo de trabalho entre APS, regulação e AE, o que pode ser feito por meio do fomento da Telemedicina e do Telessaúde, tecnologias que propiciam suporte, educação e assistência ao profissional. Além disso, a implementação de um Sistema de Prontuário Único, Telechats e reuniões capacitadoras poderia permitir o compartilhamento adequado das informações sobre as condutas médicas e a comunicação direta dos profissionais médicos entre essas três esferas do sistema de saúde. Essas ações evitariam encaminhamentos desnecessários, reduzindo filas e custos, assim como ampliando o acesso de quem realmente precisa da atenção secundária.

\section{Referências}

1. Brasil. Ministério da Saúde, Secretaria de Atenção à Saúde, Departamento de Atenção Básica. Diretrizes para o cuidado das pessoas com doenças crônicas nas redes de atenção à saúde e nas linhas de cuidado prioritárias [Ebook]. Brasília, DF: Ministério da Saúde; 2013. [acesso em 2018 maio 5]. Disponível em: http://189.28.128.100/dab/docs/geral/documento_norteador.pdf.

2. World Health Organization. World health statistics 2017: monitoring health for the SDGs, Sustainable Development Goals [internet]. Geneva: World Health Organization; 2017 [acesso em 2018 maio 10]. Disponível em: https://www.who.int/gho/publications/ world_health_statistics/2017/en/.
Espera-se que este estudo fomente novas pesquisas que indiquem alternativas para qualificar as RAS nas regiões e o cuidado do usuário na APS, a partir de melhoria na sua resolutividade.

\section{Colaboradores}

Bernardino Junior SV (0000-0002-26167680)*, Medeiros CRG (0000-0001-94660437)*, Souza CF (0000-0003-3439-4995)*, Kich J (0000-0002-8096-1770)*, Alves AM (0000-0001-6702-2739)* e Castro LC (00000001-8330-4007)* colaboraram igualmente para a elaboração do manuscrito.

3. Siqueira ASE, Siqueira-Filho AG, Land MGP. Análise do Impacto Econômico das Doenças Cardiovasculares nos Últimos Cinco Anos no Brasil. Arq. Bras. Cardiol. 2017; 109(1):39-46.

4. International Diabetes Federation. IDF Diabetes Atlas 8th Edition [internet]. Brussels: International Diabetes Federation; 2017. [acesso em 2019 mar 17]. Disponível em: https://www.idf.org/e-library/epidemiology-research/diabetes-atlas/134-idf-diabetes-atlas-8th-edition.html.

5. Rio Grande do Sul. Secretaria Estadual de Saúde. Doenças crônicas não transmissíveis [internet]. [acesso em 2018 maio 15]. Disponível em: http://bipublico. saude.rs.gov.br/index.htm.
${ }^{\star}$ Orcid (Open Researcher and Contributor ID). 
6. Brasil. Portaria $\mathrm{n}^{\circ} 2.436$, de 21 de setembro de 2017. Aprova a Política Nacional de Atenção Básica, estabelecendo a revisão de diretrizes para a organização da Atenção Básica, no âmbito do Sistema Único de Saúde (SUS). Diário Oficial da União. 21 Set 2017.

7. Brasil. Ministério da Saúde; Universidade Federal do Rio Grande do Sul. Protocolos de encaminhamento da atenção básica para a atenção especializada Endocrinologia e nefrologia [internet]. Brasília, DF: Ministério da Saúde; 2016. [acesso em 2018 abr 5]. Disponível em: http://dab.saude.gov.br/portaldab/biblioteca. php?conteudo=publicacoes/protocolos_atencao_basica_atencao_especializada.

8. Brasil. Ministério da Saúde; Universidade Federal do Rio Grande do Sul. Protocolos de encaminhamento da atenção básica para a atenção especializada Cardiologia [internet]. Brasília, DF: Ministério da Saúde; 2016. [acesso em 2018 abr 5]. Disponível em: http:// bvsms.saude.gov.br/bvs/publicacoes/protocolos_ atencao_basica_especializada_cardiologia_v_II.pdf.

9. Universidade do Rio Grande do Sul. TelessaúdeRS. Protocolos de Regulação Ambulatorial: cardiologia adulto [E-book]. Porto Alegre: UFRGS; 2017. [acesso em 2018 abr 5]. Disponível em: https://www.ufrgs.br/ telessauders/documentos/protocolos_resumos/protocolo_ses_cardiologia_20170911_v019.pdf.

10. Universidade do Rio Grande do Sul. TelessaúdeRS. Protocolos de encaminhamento para endocrinologia adulto [E-book]. Porto Alegre: UFRGS; 2018. [acesso em 2018 abr 5]. Disponível em: https://www.google. com/url?q=https://www.ufrgs.br/telessauders/documentos/protocolos_resumos/protocolos_encaminhamento_endocrinologia_revisado_TSRS_SES04jan.pd f\&sa=D\&ust=1527637756612000\&usg=AFQjCNHQd NLY3mXDf3isvlgUW7tpQJewEg.

11. Chaves LDP, Jesus BJ, Ferreira JBB, et al. Avaliação de resultados da atenção aos agravos cardiovasculares como traçador do princípio de integralidade. Saúde Soc. São Paulo. 2015; 24(2):568-577.

12. Chaves LA, Jorge AO, Cherchiglia ML, et al. Integração da atenção básica à rede assistencial: análise de componentes da avaliação externa do PMAQ-AB. Cad. Saúde Pública 2018; 34(2):e00201515.

13. Starfield B. Atenção primária: equilíbrio entre necessidades de saúde, serviços e tecnologia. Brasília, DF: Unesco; Ministério da Saúde; 2002.

14. Mendes EV. O cuidado das condições crônicas na atenção primária à saúde: o imperativo da consolidação da estratégia da saúde da família. Brasília, DF: Organização Pan-Americana da Saúde; 2012.

15. Schmitz CAA. Telessaúde como Suporte Assistencial para a Atenção Primária à Saúde no Brasil [tese]. Porto Alegre: Universidade Federal do Rio Grande do Sul; 2015; 105 p.

16. Marcolino MS, Alkmim MB, Assis TGP, et al. Teleconsultorias no apoio à atenção primária à saúde em municípios remotos no estado de Minas Gerais, Brasil. Rev Panam Salud Publica. 2014; 35(5-6):345-352.

17. Brasil. Ministério da Saúde. Secretaria de Gestão do Trabalho e da Educação na Saúde Secretaria de Gestão do Trabalho e da Educação na Saúde: políticas e ações. Brasília, DF: Ministério da Saúde; 2011.

18. Rede Nacional de Ensino e Pesquisa. Ações de telemedicina e telessaúde mudam a realidade da saúde no Brasil [internet]. 2015. [acesso em 2018 nov 18]. Disponível em: https://www.rnp.br/noticias/acoes-de-telemedicina-e-telessaude-mudam-realidade-da-saude-no-brasil.

19. Santos JS, Pereira GAJ, Bliacheriene AC, et al. Protocolos clínicos e de regulação: acesso à rede de saúde. Rio de Janeiro: Elsevier; 2012.

20. Universidade Federal do Rio Grande do Sul. RegulaSUS: Protocolos de encaminhamento 2018 [internet]. 2018. [acesso em 2018 abr 5]. Disponível em: http:// www.ufrgs.br/telessauders/nossos-servicos/teleconsultoria/regulasus.

21. Francisco HMG. Processo Assistencial Integrado do Risco Cardiovascular no Adulto. Lisboa: Direção-Geral da Saúde; 2014. [acesso em 2019 jan 15]. Dispo- 
nível em: http://nocs.pt/processo-assistencial-integrado-risco-cardiovascular-adulto/.

22. Francisco HMG. Processo Assistencial Integrado da Diabetes Mellitus tipo 2 [internet]. Lisboa: Direção-Geral da Saúde; 2013. [acesso em 2019 jan 15]. Disponível em: http://nocs.pt/processo-assistencial-integrado-diabetes-mellitus/.

23. Brandão JRM. A atenção primária à saúde no Canadá: realidade e desafios atuais. Cad. Saúde Pública. 2019; 35(J):e00178217.

24. Viana LV, Leitão CB, Kramer CK, et al. Poor glycaemic control in Brazilian patients with type 2 diabetes attending the public healthcare system: a cross-sectional study. BMJ Open. 2013; (3):e003336.

25. Fonseca JOP, Castanheira MF, Pintos SAG, et al. A importância de um centro de atenção secundária a portadores de hipertensão arterial e diabetes em um cenário para melhoria da assistência à população idosa. Rev Med Minas Gerais. 2008; 18(sup14):S25-S29.

26. Morris AJ, Burke FJT. Primary and secondary dental care: the nature of the interface. Br Dent J. 2001; 191(12):660-4.

27. Neto GCC, Antunes VH, Oliveira A. A prática da Medicina de Família e Comunidade no Brasil: contexto e perspectivas. Cad. Saúde Pública. 2019; 35(1):e00170917.

28. Anderson MIP, Demarzo MMP, Rodrigues RD. A Medicina de Família e Comunidade, a Atenção Primária à Saúde e o Ensino de Graduação: recomendações e potencialidades. Rev Bras Med Fam e Com. 2007; 3(11):157-172.

Recebido em 13/08/2019

Aprovado em 10/06/2020

Conflito de interesses: inexistente

Suporte financeiro: Universidade do Vale do Taquari (Univates) 